\title{
Entwicklung eines Produktarchitekturmodells zur Ableitung modularer Produktstrukturen
}

Jan Küchenhof ${ }^{1}$, Dieter Krause ${ }^{1}$

${ }^{1}$ Institute of Product Development and Mechanical Engineering Design (PKT), Hamburg University of Technology (TUHH), Germany

\section{Abstract}

Modularization can help shorten product development lead-times, enable customer-oriented product differentiation, enable economies of scale through commonality and economies of scope such as innovativeness and flexibility. Modular product structures based on modular product architectures are to be developed in order to make these advantages available to companies within product family creation. This contribution provides methodical support in conceptualization of the initial product family by adapting methodical steps and graphical product development models. This forms the basis for future product generation design and efficient product life-cycle management.

Keywords: New Product Development, Product Architecture Design, Design for Variety, Modularization

\section{Einleitung und Motivation}

Kürzere Produktlebenszyklen, globaler Wettbewerb und neue Technologien setzen Unternehmen zunehmend unter Innovationsdruck [7]. Die stark fragmentierten Märkte erfordern hierbei kundenangepasste Produktlösungen. Die Neuentwicklung von Produkten ist eine Möglichkeit das Produktprogramm auszuweiten und innovative Lösungen in wachsenden Märkten zu platzieren [10]. 
Um die varianteninduzierte Produkt- und Prozesskomplexität unter Beibehaltung der externen Angebotsvielfalt zu reduzieren, eignen sich modulare Produktfamilien, dessen Entwicklung durch den Methodenbaukasten des Integrierten PKT-Ansatzes zur Entwicklung modularer Produktfamilien (PKT-Ansatz) unterstützt wird [8]. Mit verschiedenen Zielsetzungen, wie beispielsweise der Komplexitätsreduzierung, Konfiguration statt Konstruktion oder hohe Kommunalität wird der Ansatz kontinuierlich weiterentwickelt. Die bekannten Vorteile der vielfaltsorientierten Entwicklung modularer Produktstrukturen sollen der Produktentwicklung von Beginn an zugänglich gemacht werden. Dies soll zudem die Grundlage für die Strukturierung von Organisations- und Prozessstrukturen bieten und das Wissensmanagement über Komponenten und ihre Interaktionen bereits in der frühen Phase nachhaltig unterstützen. Unter dieser Zielsetzung soll der PKT-Ansatz im Kontext der Produktentstehung erweitert werden. Die frühe Phase der Produktentwicklung kann in die drei Phasen Chancenidentifikation und Markt-/ Technologieanalyse, Produktkonzept und -definition und Projektplanung unterteilt werden [5]. Mit dem letzten Schritt werden weitere Entwicklungstätigkeiten wie der Prototypenbau und -test, Produktion und Markteinführung eingeleitet. [5] Die Konzeptfindung der initialen Produktfamiliengestaltung soll mithilfe geeigneter Methoden und Produktentwicklungsmodelle unterstützt werden. Es ist angestrebt die Effekte der Hebelwirkung modularer Produktstrukturen dazu zu nutzen, Produktentwicklungszeiten zu verkürzen, eine kundengerechte Produktdifferenzierung zu ermöglichen, Skaleneffekte durch Kommunalität zu schaffen und Verbundeffekte wie die Innovationsfähigkeit und Flexibilität zu stärken [11].

\section{Forschungsproblem und Forschungsziel}

Traditionelle Methoden zur Entwicklung neuer Produkte ((NPD, engl.: New Product Development) fokussieren zumeist die Entwicklung einzelner, integraler Produktvarianten. Die Produktarchitektur ist das Ergebnis von sequentiellen Konstruktions- und Entwicklungsprozessen [14]. Modulare Produktstrukturen eignen im Umgang mit varianteninduzierter interner Produkt- und Prozesskomplexität [8], werden allerdings zumeist im Kontext gewachsener Unternehmens- und Produktstrukturen betrachtet. Sanchez [14] beschreibt wie eine modulare Produktentwicklung die Koordination von Wissen, die Schaffung organisatorischer Strukturen und die Kollaboration mit Entwicklungspartnern und Zulieferern unterstützen kann [14]. Die Integration verschiedener Interessengruppen im Rahmen der NPD [2] sowie die gemeinsame Betrachtung von NPDund Globalisierungsstrategien [7] ist Fokus aktueller Forschung - der Einfluss von Produktvielfalt und Modularisierungsstrategien wird allerdings nicht betrachtet. Die Wahl der Schnittstellen der Komponenten innerhalb der entwickelten Produktarchitektur kann die Innovationsfähigkeit und Reaktionsfähigkeit 
von Unternehmen erheblich beeinflussen und ist somit kritisch für die strategische Geschäftsentwicklung [10]. Ziel ist es die Entwicklung modularer Produktarchitekturen zu unterstützen, um modulare Produktstrukturen abzuleiten und schließlich modulare Produkte in Form von Prototypen und Produktvarianten über Generationen hinweg in diesem Rahmen zu entwickeln. Hierfür müssen sowohl geeignete Entwicklungsmethoden sowie Produktentwicklungsmodelle bereitgestellt werden.

\section{Stand der Wissenschaft}

Im Stand der Wissenschaft wird zunächst auf die essentiellen Unterschiede zwischen traditioneller und modularer Produktentwicklung eingegangen. Die variantengerechte Produktgestaltung (DfV, engl.: Design for Variety) nach Kipp wird im Detail vorgestellt, da es die traditionellen Ansätze um den Aspekt der Vielfalt ergänzt und gut erprobte Entwicklungsmodelle beinhaltet [8]. Die Vorteile von Modularisierung im Rahmen des organisatorischen Lernens, der erhöhten Innovationsfähigkeit und der Reduzierung der Entwicklungszeiten werden kurz beschrieben. Zunächst erfolgt eine Definition des Begriffs Produktarchitektur, da dies zum Verständnis sowohl der traditionellen als auch modularer Entwicklungsvorgehen ist.

\subsection{Definition von Produktarchitekturen}

Die gängige Definition der Produktarchitektur nach Ulrich [16] ist die Anordnung funktionaler Elemente, die Zuordnung funktionaler Elemente zu physischen Komponenten und die Spezifikation der Schnittstellen zwischen interagierenden physischen Komponenten beschrieben. Es wird unterschieden zwischen modularen Architekturen, die eine 1:1-Zuordnung von funktionalen Elementen auf die physikalischen Komponenten des Produkts umfasst und entkoppelte Schnittstellen zwischen den Komponenten spezifiziert und integralen Architekturen, die eine komplexe (nicht 1:1-) Zuordnung von Funktionselementen auf physikalische Komponenten und/oder gekoppelten Schnittstellen zwischen Komponenten beinhalten. [16] Eine integrale Produktarchitektur eignet sich bei der Zielsetzung hoher Produktleistung und niedriger Produktionskosten. Eine modulare Produktarchitektur bei der Fokussierung langfristig niedriger Entwicklungskosten und kurzen Entwicklungszeiten. [12]

\subsection{Konventionelle vs. Modulare Produktentwicklung}

Der konventionelle Produktentwicklungsprozess beginnt mit einer umfangreichen Marktstudie zur Identifikation spezifischer Charakteristika zur Erfüllung bestimmter Kundenbedürfnisse. Diese werden der Produktentwicklung 
übergeben, die unter bestimmten Rahmenbedingungen wie Kosten und Leistung versucht, ein optimales Produkt zu gestalten. Die Produktfunktionalität wird den Komponenten früh zugeordnet, die Schnittstellen zwischen den Komponenten werden allerdings erst während der Komponentenentwicklung festgelegt. Das Ergebnis des optimierten Produktdesigns im traditionellen Entwicklungsprozess ist eine entsprechend komplexe Produktarchitektur. Komponenten oder Schnittstellenänderungen während und nach der Entwicklung betreffen hierdurch das komplette Produkt, wodurch die Umgestaltung zeitaufwändig und kostenintensiv ist.

Die modulare Produktentwicklung hingegen folgt der Entwicklungsregel, dass Wechselwirkungen zwischen Komponenten minimiert werden. Die frühe Spezifikation der Schnittstellen zwischen den Komponenten erlaubt es, die Komponenten in einem gewissen Variationsspektrum anzupassen, um Produktvarianten mit verschiedenen Funktionalitäten, Eigenschaften oder Leistungsklassen zu entwickeln. Hierfür muss die Produktarchitektur am Anfang des Entwicklungsprozesses, abgestimmt auf einen bestimmten Satz von Produkteigenschaften definiert werden. Resultat des Entwicklungsprozesses ist eine modulare Produktarchitektur. Diese flexible Plattform ermöglicht einfache Produktvariationen anhand eines Basisdesigns und ist ein wichtiger Faktor für weitere strategische Maßnahmen wie die Organisation des Entwicklungsprozesses oder die Strukturierung von Supply-Chain- und Montagestrukturen mit Hilfe von Postponement-Strategien. [14]

\subsection{Organisatorische Struktur= und Lerneffekte}

Produktarchitekturen bestimmen zum großen Teil die Wissensstruktur eines Unternehmens sowie die Organisation des Entwicklungsprozesses. Anstatt der traditionellen, sequentiellen Durchführung von Gestaltungs- und Entwicklungsaufgaben, können diese Tätigkeiten bei der modularen Entwicklung komponentenweise parallel durchgeführt werden und eine komplette Informationsinfrastruktur über das Produkt wird geschaffen. [14] Die frühe Definition der Schnittstellen ermöglicht die Koordination einer fragmentierten Organisationsstruktur; Entwicklungspartner und Zulieferer können gezielt bei der Komponentenentwicklung einbezogen werden.

Das Lernen über die Komponenten und ihre Interaktion kann in vier Moden des Lernens eingeteilt werden [15] (Bild 1, links). Modulare Produktarchitekturen verbessern das Lernen vom inkrementellen Komponentenlevel bis zum radikalen Architektur- und Komponentenlevel. Dieses zusätzliche Wissen kann genutzt werden, um den Rahmen der architekturellen Innovation [4] (Bild 1, rechts) nachzuvollziehen und die Innovationsstrategie entsprechend 
auszurichten. Die schnelle Anpassung einzelner Komponenten auf sich verändernde Marktbedürfnisse und die gezielte Umgestaltung von Schnittstellen ermöglichen Flexibilität und innovative Produktkonzepte. Durch diese strukturellen Änderungen von Kernkonzepten und Komponenten-Schnittstellen können dominierende Designs aus dem Markt verdrängt werden. [4]
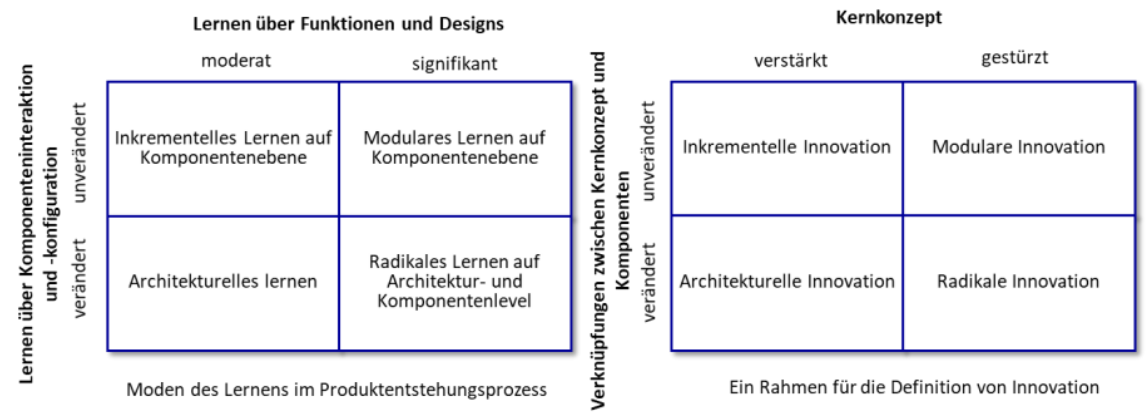

Bild 1: Links: Moden des Lernens [15] Rechts: Ein Rahmen für die Definition von Innovation [4] (eigene Darstellung)

\subsection{Kernschritte des Integrierten PKT-Ansatzes zur Entwicklung mo- dularer Produktfamilien}

Zur Entwicklung modularer Produktfamilien eignen sich die DfV nach Kipp [6] und die anschließende produktstrategische Lebensphasenmodularisierung nach Blees [1] als Kernschritte des PKT-Ansatzes [8]. Bei der DFV finden die grundlegenden Schritte der Produktentwicklung Anwendung, weshalb es für die generelle Entwicklung von Produkten geeignet ist [3]. Der im Pahl und Beitz [13] beschriebene Entwicklungsprozess unterstützt mit entsprechenden Produktentwicklungsmodellen die Bereiche der Anforderungen, Funktionen, Wirkprinzipien und Komponenten. Auch das Variety Allocation Model(VAM) als zentrales Entwicklungsmodell der DfV unterstützt diese Entwicklungsebenen mit entsprechenden Abstraktionsschritten [3]. Die Anforderungen werden aufgenommen und in kundenrelevanten, differenzierenden Eigenschaften (krE) ausgedrückt. Diese werden anhand ihrer Ausprägungen kombiniert und in einem Vielfaltsbaum (TeV, engl.: Tree of external Variety) strukturiert, um die externe Vielfalt abzubilden. Die krE bilden die erste der insgesamt vier Ebenen des VAM. Die Ebene der Funktionen wird in Form der umsatzorientierten Produktfamilienfunktionsstruktur (PFFS) aufgenommen. Die zur Eigenschaftsrealisierung notwendigen Funktionen werden nun ebenfalls in VAM übertragen. Die zur 
Erfüllung der Funktionen verwendeten, varianten Wirkprinzipien und -elemente werden auf der dritten Ebene eingetragen. Im Rahmen der Analyse oder Optimierung von Produktfamilien können variante Wirkprinzipien und -elemente oder variante technische Merkmale zur Betrachtung herangezogen werden [3]. Die Komponenten und Schnittstellen werden im Module Interface Graph eingetragen und identifizierte variante und optionale Komponenten in die unterste Ebene des VAM übernommen. Die benachbarten Modellebenen werden nun beginnend von der ersten kausal miteinander verknüpft. Wenn beispielsweise eine differenzierende Eigenschaft eine funktionale Varianz verursacht, werden diese Elemente verbunden; ist ein Wirkprinzip zur Erfüllung einer funktionalen Vielfalt erforderlich, werden diese verknüpft, etc. (Bild 2, a).Das von Kipp definierte Idealbild einer variantengerechten Produktfamilie folgt den vier Kriterien der: Differenzierung in Standard- und Variantenkomponenten, Reduzierung zum Träger einer kundenrelevanten, differenzierenden Eigenschaft, 1:1-Zuordnung von differenzierenden Eigenschaften zu Variantenkomponenten und Entkopplung der Variantenkomponenten. [6], [8]

Die Lebensphasenmodularisierung sieht als Eingangsgröße die variantengerechten Komponenten der DfV vor. Neben einer technisch-funktionalen Modularisierung wird die Produktfamilie produktstrategisch modularisiert. Einflussgrößen der Produktlebensphasen (Produktentwicklung, Beschaffung, Herstellung, ...) werden aufgenommen und sind in Netzplänen als Modultreiber mitsamt -ausprägungen repräsentiert. Der Einfluss auf die Komponenten wird identifiziert und lebensphasenspezifische, „Wunsch"-Modulstrukturen werden gebildet. Die modulare Lebensphasenstruktur wird im Modular Process Chart visualisiert und in Abstimmung der Produktlebensphasen harmonisiert. Leitlinie der Harmonisierung ist, dass Module zur Steigerung der Wertschöpfung bis zur vollständigen Zusammensetzung des Produktes nicht kleiner, sondern größer werden. [1], [8]

\section{Anpassung von Vorgehen und Modell mit anschließender Durchfüh- rung im fiktionalen Fallbeispiel}

Da der PKT-Ansatz auf gewachsenen Produktfamilien beruht, bedarf die neue Zielsetzung einiger Anpassungen. Die identifizierten Handlungsbedarfe werden im Folgenden kurz aufgezeigt und das eigene Vorgehen erläutert. 


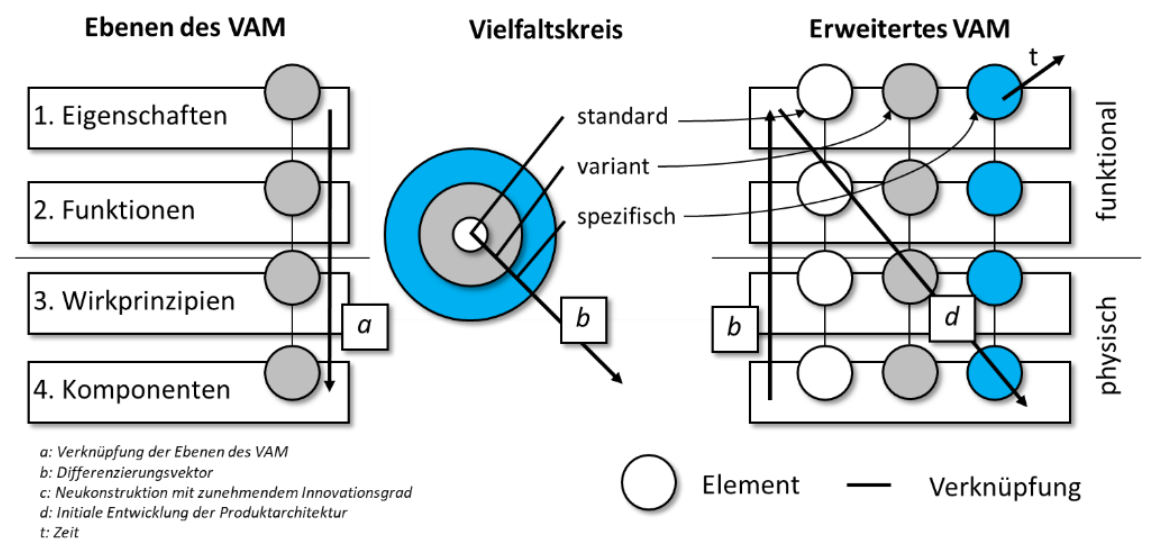

Bild 2: Aufbau des VAM und Ergänzungen im Kontext der Neuentwicklung

\subsection{Stufen der Vielfalt}

Zur Fokussierung der Vielfalt übernimmt Kipp in die Ebene der krE nur jene auf, die zwischen Kunden oder verkauften Produktvarianten differenzieren. Bei NPD ohne eigene Vorgängerprodukte existiert zunächst keine Angebotsvielfalt aus vergangenen Geschäftsperioden. Daher ist es notwendig zunächst alle krE betrachten und zu klassifizieren. Ob diese über die Zeit variieren sollte früh festgestellt werden und kann zum Teil Resultat von Iterationsschleifen während der Entwicklung sein. Zudem können Teile des Basisangebotes für den Kunden essentielle Bestandteile der Kernkompetenzen des Unternehmens repräsentieren und benötigen eine besondere Betrachtung, da sie eine zentrale Rolle bei der Entwicklung einnehmen. Standardanteile werden in den Entwicklungsmodellen weiß dargestellt, differenzierende sind grau gekennzeichnet. Eine zusätzliche Stufung wird zur Betrachtung und leichten Identifikation potentieller kundenspezifischer Elemente als besondere Form der Varianz eingebracht; diese Anteile erhalten eine blaue Färbung. Die Überlagerung der Vielfaltsstufen resultierend in dem Vielfaltskreis (Bild 2, Mitte) gibt die Leitlinie der Entwicklung vor - mit dem Kredo der Standardisierung nach innen und der Differenzierung nach außen. Eigenschaften und Komponenten sollen nur dort variieren wo es technisch notwendig ist oder für den Kunden spürbar wird. Dies wird durch den Differenzierungsvektor indiziert (Bild 2, b). Da die Produktvielfalt über die Zeit ansteigt, muss dies bei der Entwicklung berücksichtigt werden und ist hier mit dem Zeitvektor $t$ gekennzeichnet. Die Standard-, Varianten- und spezifischen Elemente können in jeder Abstraktionsebene Verwendung finden und werden dementsprechend ins erweiterte VAM integriert (Bild 2, rechts). 


\subsection{Anpassung der Kernschritte des PKT-Ansatzes}

Im Kontext der Neuentwicklung werden einige Methodenschritte des Integrierten PKT-Ansatzes angepasst. Die Erfassung der externen Vielfalt wird aufgrund fehlender eigener Produkte als Planung der externen Vielfalt bezeichnet. Auch beim Methodenschritt Erfassung der internen Vielfalt muss aufgrund fehlender interner Strukturen der Planungsaspekt hervorgehoben werden und wird mit dem Schritt Planung der internen Vielfalt berücksichtigt. Die Schritte der Zielfindung, Konzeptfindung der DfV und der Konzeptauswah/ bleiben bestehen. Die variantengerechten Komponenten können anschließend in die produktstrategische Modularisierung überführt werden. Es sollte möglich sein den Produktlebensphasenprozess früh in eine abgestimmte Form zu bringen, wird in diesem Beitrag allerdings nicht fokussiert. Eine wichtige Anpassung des Modularisierungsvorgehens wird jedoch bereits an dieser Stelle vorgeschlagen. Die technisch-funktionale Modularisierung nach den Heuristiken kann bereits während der DfV durchgeführt werden, da mit der flussorientierten PFFS bereits auf der zweiten Abstraktionsebene eine geeignete Struktur hierfür vorliegt. Die Lösungsfindung auf Ebene der Wirkprinzipien kann durch die Vorgabe funktionaler Blöcke gut strukturiert und geleitet werden.

\subsubsection{Darstellung von drei Produktgenerationen im erweiterten VAM}

Im Folgenden wird die Methode im Rahmen der NPD anhand dreier fiktionaler Produktgenerationen mit unterschiedlicher Variantenvielfalt simuliert und im erweiterten VAM dargestellt. In diesem Fall wird eine abgeschlossene Planung der externen Vielfalt vorausgesetzt. Folgend der Definition, dass Produktvarianten gleichzeitig verkauft werden und bei einem Generationswechsel die vorherige Produktgeneration nicht mehr am Markt angeboten wird, ist eine fiktive externe Angebotsvielfalt über drei Produktgenerationen im $\mathrm{TeV}$ in Bild 3 dargestellt. Die Eigenschaften $(E)$ in der obersten Ebene sind spaltenweise mit den jeweiligen Ausprägungen (A1.x-A5.x) angeordnet. Die Strukturierung der Eigenschaften erfolgt nach dem Maß der Angebotsvielfalt aufsteigend nach rechts. Firmenseitig (links) sollen kommunale Anteile zur Kostensenkung angestrebt werden, während angebotsseitig (rechts) eine ausreichende Produktdifferenzierung berücksichtigt werden muss. Die Ausprägungen werden zu je einer Produktvariante verknüpft (V1.1-V3.6). Die Produktgenerationen (G1-G3) sind visuell durch drei Ebenen getrennt. G1 besteht aus einer Standardvariante, die als Minimum Viable Product schnell auf den Markt gebracht werden kann. In G2 werden zwei Eigenschaften in neuen, varianten Ausprägungen angeboten, z.B. im Zuge eines Facelifts des Produktes. In G3 wird ein Technologieupgrade der Kernfunktionen durchgeführt, so dass E1 und E2 in verbesserter Ausführung (A1', A2') angeboten werden können. Zudem wird identifiziert, dass 
für krE E5 eine hohe Differenzierung gewünscht ist, weshalb mit A5.3 eine kundenspezifische Ausprägung angeboten wird.

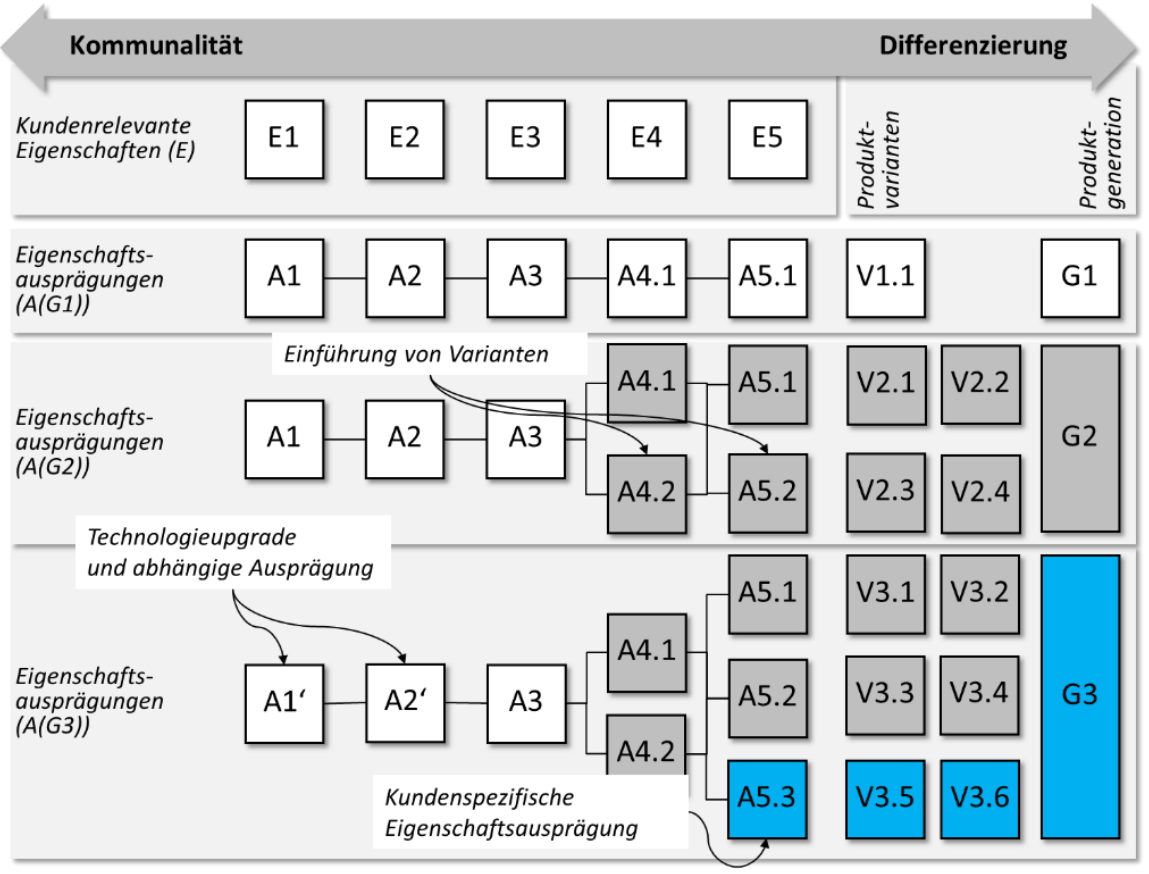

Bild 3: Fiktive externe Angebotsvielfalt über drei Produktgenerationen im angepassten $\mathrm{TeV}$

Auf Basis der externen Vielfalt wird die DfV durchgeführt. Die Elemente der vier Abstraktionsebenen werden gemäß der DfV nach Kipp ins VAM übertragen und dort verknüpft (Bild 2, d) (Bild 4). Es wird idealisiert angenommen, dass für jede Produkteigenschaft (E1-E5) genau ein funktionaler Block (F1-F5) entwickelt werden muss, der durch eine technische Lösung mittels eines Lösungsprinzips (W1-W5) durch eine Komponente (K1-K5) realisiert wird, um eine künstliche 1:1-Zuordnung zwischen Eigenschaften und Komponenten zu erreichen. Die zeitliche Erweiterung des Modells durch die Produktgenerationen G2 und G3 wird durch eine jeweilige Ebene hinter G1 dargestellt. Zur Identifikation und Nachvollziehbarkeit der notwendigen Änderungen werden nur die Differenzen zwischen den Ebenen aufgetragen. Durch die Erweiterung des Angebotes werden die bestehenden Eigenschaften beeinflusst und die betroffenen Entwicklungsstränge werden sequentiell durchlaufen, um die Erweiterung des Angebotes intern umzusetzen. Mögliche Änderungen sind z.B. die Anpassung der 
Funktionalität, die Variation eines Wirkprinzips oder die Änderung oder Substitution von Komponenten. In G2 werden nur Strang 4 und 5 verändert - die neue Angebotsvielfalt durch die eingeführte Variante wird durch die graue Färbung des Elements deutlich. Sollte eine Varianz bzgl. Funktionen, Wirkprinzipien oder Komponenten zur Erfüllung der neuen krE notwendig sein, werden diese Elemente ebenfalls grau gefärbt. In G3 ist der Technologiesprung durch den neuen Standard erkennbar, da die Stränge 1 und 2 weiß bleiben - jedoch eine Änderung durch alle Abstraktionsebenen hinweg notwendig ist. Die Einführung des kundenspezifischen Angebotes in G3 ist durch die blaue Färbung leicht ersichtlich.

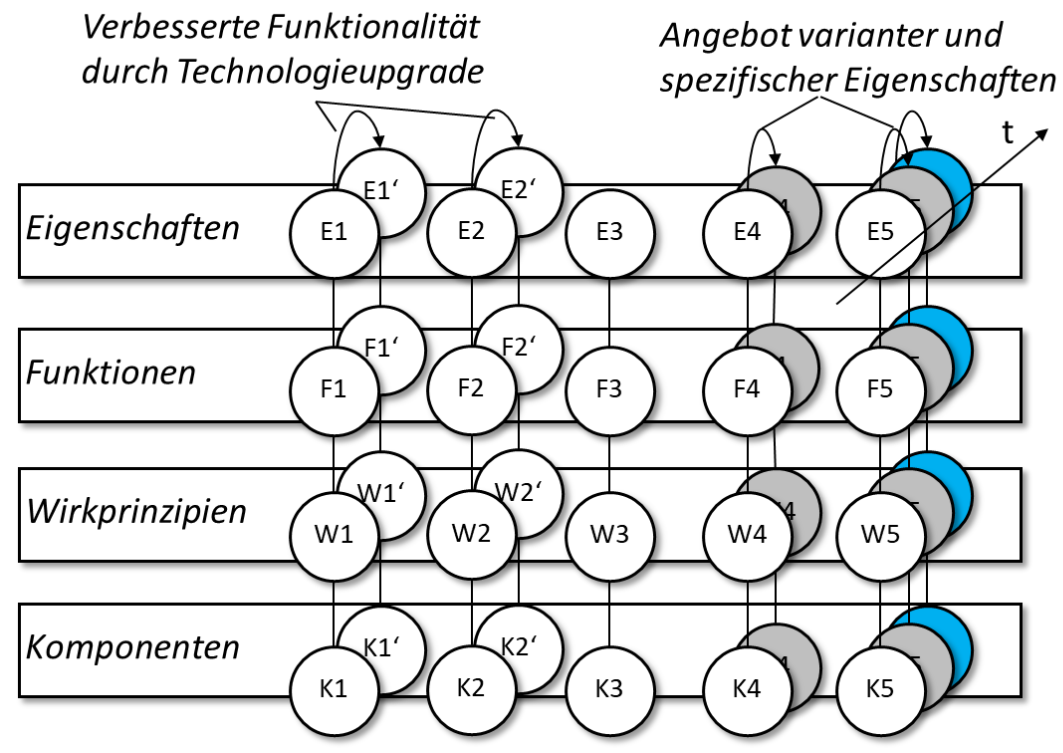

Bild 4: Erweitertes VAM mit Darstellung der drei fiktiven Produktgenerationen

\section{Ergebnisse und Diskussion}

Das VAM wurde hinsichtlich der Standardanteile erweitert und die Vielfalt in variante und spezifische Anteile zergliedert. Die Änderung von Elementen im VAM kann über Produktgenerationen hinweg nachverfolgt werden, indem dem Modell zusätzliche zeitliche Ebenen hinzugefügt werden und nur die von Änderungen betroffenen Elemente dargestellt werden. Durch die 1:1-Zuordnung von Eigenschaften, Funktionen, Wirkprinzipien und Komponenten wird ein Teil der Produktarchitektur abgebildet werden. Komponentenänderungen können separat vorgenommen und neue Eigenschaften im Rahmen des definierten 
Entwicklungsrahmen angeboten werden, sofern die Schnittstellen zwischen den Komponenten entsprechen überdimensioniert sind. Bei der Lösungsfindung im VAM können schrittweise Lösungsansätze mit höheren Verbesserungspotentialen (und dementsprechend höherem Risiko und Entwiclkungsaufwand), beginnend von der Ebene der varianten Komponenten bis hin zu den Funktionen, erarbeitet werden (Bild 2, c). Während Kipp die Ebenen 2-4 betrachtet und entsprechend unterstützt, sollte bei der NPD auch die erste Ebene mit höchstem Innovationspotential betrachtet werden. Diese Ebene ist kritisch, da die Produkteigenschaften sich über die Zeit verändern und die Produktarchitektur über die Zeit and die Marktbedürfnisse angepasst oder erneuert werden muss.

Das vorgestellte methodische Vorgehen soll weitergehend detailliert werden und die angepasste DfV soll in einem realen Fallbeispiel Anwendung finden. Außerdem soll untersucht werden, wie der Produktlebensphasenprozess mit ideal variantengerechten Komponenten initial strukturiert werden könnte. Im Kontext der Produkt- und gleichzeitiger Geschäftsentstehung sind alle weiteren Lebensphasen von hoher Unsicherheit betroffen und bedürfen einer strukturierten Organisation und Planung, um eine effiziente und nachhaltige Produktentwicklung sicher zu stellen.

\section{Literatur}

[1] Blees, C., "Eine Methode zur Entwicklung modularer Produktfamilien", Dissertation, Institut für Produktentwicklung und Konstruktionstechnik, Technische Universität Hamburg, Hamburg, 2011.

[2] Driessen, P. H., Hillebrand, B., "Integrating Multiple Stakeholder Issues in New Product Development: An Exploration", Journal of product innovation Management 30, 2013, pp. 364-379.

[3] Gebhardt et al., "Nutzung von "Merkmalen" und "Eigenschaften" zur Beschreibung und Analyse von Produktvarianz", Design for X - Beiträge zum 23. DfX-Symposium, Bamberg, 2012, pp.175-186.

[4] Henderson, R., Clark, K. B., "Architectural Innovation: The Reconfiguration of Existing Product Technologies and the Failure of Established Firms", Administrative Science Quarterly, 36, 1990, pp. 9.30.

[5] Khurana, A., Rosenthal, S., "Integrating the Fuzzy Front End of New Product Development", Sloan Management Review, 1997, pp. 103-120. 
[6] Kipp, T., "Methodische Unterstützung der variantengerechten Produktgestaltung", Dissertation, Institut für Produktentwicklung und Konstruktionstechnik, Technische Universität Hamburg, Hamburg, 2012.

[7] Kleinschmidt et al., "Performance of Global New Product Development Programs: A Resource-Based View", Journal of Product Innovation Management 24, 2007, pp. 419-441.

[8] Krause, D., Gebhardt, N., "Methodische Entwicklung modularer Produktfamilien - Hohe Produktvielfalt beherrschbar entwickeln", Springer, Berlin, 2017.

[9] Mikkola, J. H., „Product Architecture Design: Implications for Modularization and Interface Management", LINK Workshop: Organizing Processes of Learning, Copenhagen, 2000.

[10] Mikkola, J. H., Gassmann, O., "Managing Modularity of Product Architectures: Toward an Integrated Theory", IEEE Transactions on Engineering Management, Vol. 50 No. 2, 2003, pp. 204-218.

[11] Mikkola, J. H., "Capturing the Degree of Modularity Embedded in Product Architectures", Journal of Product Innovation Management 23, 2006, pp. 128-146.

[12] Müller, M., „Management der Entwicklung von Produktplattformen”, Dissertation, Hochschule für Wirtschafts-, Rechts und Sozialwissenschaften, Universität St. Gallen, St. Gallen, 2000.

[13] Pahl, G., Beitz, W. et al.: „Konstruktionslehre, Methoden und Anwendung erfolgreicher Produktentwicklung", Springer-Verlag, Berlin, 2013.

[14] Sanchez, R., "Strategic Product Creation: Managing New Interactions of Technology, Markets, and Organizations", European Management Journal, Elsevier, Vol. 14 No. 2, 1996, pp. 121-138.

[15] Sanchez, R., Mahoney, J., "Modularity, Flexibility, and Knowledge Management in Product and Organization Design", Strategic Management Journal, Vol. 17, 1996, S. 63-76.

[16] Ulrich, K.; "The role of product architecture in the manufacturing firm", Research Policy 24, 1995, pp. 419-440. 